\title{
L'angiogenèse tumorale
}

L'angiogenèse est un processus essentiel lors de tout événement de réparation ou de prolifération tissulaire, normale ou tumorale. Elle requiert la migration, la multiplication et la différenciation de cellules endothéliales issues des vaisseaux préexistants. Une très grande diversité de polypeptides doués de propriétés angiogéniques est maintenant connue ; certains sont surtout actifs sur l'activité mitotique des cellules endothéliales, d'autres sur leur différenciation. L'angiogenèse étant indispensable à la croissance tumorale, toute substance antiangiogénique, naturelle ou artificielle, a un potentiel antinéoplasique. Le produit d'un anti-oncogène présomptif serait ainsi un inhibiteur de l'angiogenèse. Des composés, telles les héparines à bas poids moléculaire, et des stérö̈des ont, notamment en association, des propriétés anti-angiogéniques et constituent ainsi une intéressante voie de recherche thérapeutique.

\section{Gérard Tobelem}

\section{ADRESSE}

G. Tobelem : professeur d'hématologie à l'université Paris VII. Institut des vaisseaux et du sang, hôpital Lariboisière, 8 , rue Guy-Patin, 75010 Paris, France. e système vasculaire assure l'acheminement du sang indispensable à la vie cellulaire, au niveau de chaque tissu. Sur plus de $50 \mathrm{~km}$, le système vasculaire est tapissé intérieurement par une monocouche de cellules endothéliales dont la surface totale est supérieure à $6500 \mathrm{~m}^{2}$. Plus de $90 \%$ de cette surface concernent la microcirculation qui joue donc un rôle considérable dans les échanges gazeux, plasmatiques et cellulaires entre sang et tissus. Dans les conditions normales, l'endothélium est soumis à un très faible renouvellement cellulaire, qui se mesure en terme d'années. A l'état physiologique, les seules situations qui s'accompagnent d'une angiogenèse, c'est-àdire d'une croissance vasculaire, sont l'embryogenèse et la formation du placenta, la croissance générale et les cycles menstruels. En cas de répara- tion tissulaire après traumatisme, une angiogenèse peut également se produire. La formation de néovaisseaux peut donc nécessiter une croissance de cellules endothéliales proche de celle de certaines cellules à renouvellement rapide, comme les cellules hématopoïétiques par exemple. Cette formation de néovaisseaux peut être particulièrement importante dans certains états pathologiques, c'est le cas de l'angiogenèse tumorale, qui joue un rôle crucial dans le développement des tumeurs et leurs métastases.

\section{Les différentes étapes de l'angiogenèse}

Plusieurs modèles expérimentaux d'étude de l'angiogenèse in vitro ou in vizo ont été développés (pour revue 
voir [1]). Quatre techniques ont été particulièrement étudiées : (1) micropoche cornéenne avec implantation d'un facteur purifié ou d'un extrait non purifié (broyat de cellules ou de tissu) dans la cornée d'un lapin, d'une souris ou d'un rat ; (2) inclusion de substances angiogéniques dans un polymère biocompatible implanté chez un animal ; (3) membrane chorioallantoïque de l'embryon de poulet ; (4) culture in vitro de cellules endothéliales humaines ou animales provenant de macro- ou de microcirculation. Grâce à ces modèles, plusieurs informations ont pu être enregistrées (figure 1).

- La séquence des événements est pratiquement identique quel que soit le stimulus (immunologique, inflammatoire, tumoral ou de réparation). Les néocapillaires vont se former à partir des capillaires normaux ou des veinules post-capillaires. Les cellules endothéliales émettent des pseudopodes qui envahissent la membrane basale, puis elles migrent par paires à travers le passage [2].

- Les premières étapes sont indépendantes de la prolifération des cellules endothéliales car elles sont encore observées après irradiation du foyer exploré [3].

- La présence maintenue du stimulus angiogénique est nécessaire. Si ce stimulus est extrait du foyer angiogénique, les néocapillaires régressent puis disparaissent [4].

- L'angiogenèse peut être induite par des stimuli directs (facteurs de croissance et angiogéniques, sur lesquels nous reviendrons) ou par des stimuli indirects capables d'agir sur d'autres cellules (macrophages par exemple) en les activant et en leur faisant sécréter des facteurs de croissance et angiogéniques [5].

- La matrice vasculaire joue un rôle très important (figure 2, page suivante). Collagène de type IV, fibronectine et laminine sont très vraisemblablement impliqués dans la migration des cellules endothéliales et dans leur organisation en tubes [6-8]. Il a été récemment montré que les séquences

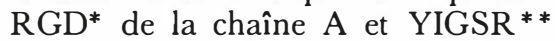
de la chaîne B de la laminine étaient indispensables pour la formation des

\footnotetext{
* $R$ : arginine $G$ : glycine. $D$ : acide aspartique est celle qui concerne la néovascula** $Y$ : tyrosine; $I$ : isoleucine; $S$ : sérine. risation tumorale [12].
} et prolifération. tubes capillaires [9]. La dégradation de certains composants de la matrice est un autre événement important. Cette dégradation permet, d'une part, l'invasion du tissu matriciel par les cellules endothéliales qui migrent et, d'autre part, la libération des facteurs de croissance qui peuvent être stockés par les glycosaminoglycanes. Les enzymes responsables de la digestion matricielle sont des protéases (plasmine, métalloprotéases, collagénases) ou des héparanases [10, 11].

- L'angiogenèse peut s'observer dans de nombreuses affections ophtalmologiques (rétinopathie proliférative diabétique, par exemple), dermatologiques (psoriasis), immunologiques et inflammatoires (polyarthrite rhumatoïde) ou dégénératives (plaque d'athérosclérose). Mais l'angiogenèse la plus intense et la plus persistante

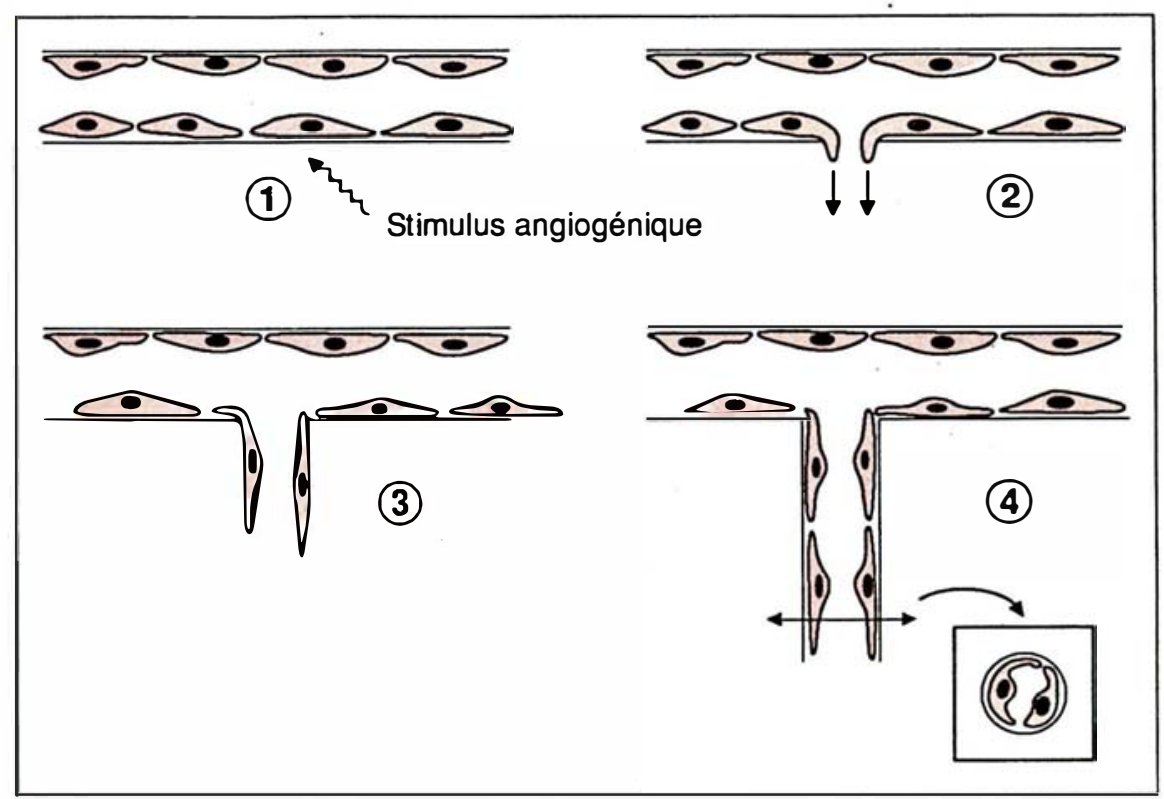

Figure 1. Principales étapes de l'angiogenèse. 1. Capillaire soumis à une stimulation angiogénique. 2. Début de migration des cellules endothéliales à travers la membrane basale. 3. Migration, élongation et différenciation des cellules endothéliales. 4. Formation des tubes capillaires dans l'espace (encadré)

L'angiogenèse comporte donc trois étapes - migration, différenciation et prolifération - intégrées dans le temps et dans l'espace par une série de signaux encore incomplètement connus. Les mécanismes moléculaires de la formation des tubes, par exemple, qui est une étape fondamentale de l'angiogenèse, ne sont pas encore élucidés. Ingber et Folkman [13] ont formulé le concept d'" intégrité tensionnelle " selon lequel les signaux nécessaires aux modifications phénotypiques seraient induits par des forces de tension exercées par des composants de la matrice extracellulaire.

\section{La néovascularisation tumorale}

Deux principes fondamentaux aujourd'hui bien établis sont à la base de la compréhension de l'angiogenèse tumorale : 


\section{RÉFÉRENCES}

1. Folkman J, Kalgsbrun M. Angiogenic factors. Science 1987; $235: 442-7$.

2. Ausprunk DH, Folkman J. Migration and proliferation of endothelial cells in preformed and newly formed blood vessels during tumor angiogenesis. Microvasc Res $1977 ; 14: 53-65$

3. Sholley MM, Ferguson GP, Seibel HR, Montour HR, Wilson JD. Mechanisms of neovascularization : vascular sprouting can occur without proliferation of endothelial cells. Lab Invest 1984; 51 : 624-34.

4. Ausprunk D, Falterman K, Folkman J. The sequence of events in the regression of corneal capillaries. Lab Invest 1978; 38 : 284-94.

5. Polverini PJ, Leibovich SJ. Induction of neovascularization in vivo and endothelial proliferation in vitro by tumor-associated macrophages. Lab Invest 1984; 51 : 635-42.

6. Furcht LT. Critical factors controlling angiogenesis : cell products, cell matrix and growth factors. Lab Invest 1986 ; 55 : 505-9.

7. Dejana E, Colella S, Conforti G, Abbadini M, Gaboli M, Marchisio PC. Fibronectin and vitronectin regulate the organization of their respective Arg-Gly-Asp adhesion receptors in cultured human endothe lial cells. J Cell Biol 1988 ; 107 : 1215-23.

8. Ingber DE, Madri JH, Folkman J Endothelial growth factors and extracellular matrix regulate DNA synthesis through modulation of cell and nuclear expansion. In Vitro $1988 ; 23: 387-94$.

9. Grant D, Tashiro KI, Segui-Real B, Yamada Y, Martin G, Kleinman H. Two different laminin domains mediate the differenciation of human endothelial cells into capillary-like structures. Cell $1989 ; 58$ : 933-43.

10. Mignatti P, Tsuboi R, Robbins E, Rifkin $\mathrm{D}$. In vitro angiogenesis on the human amniotic membrane : requirement for basic fibroblast growth factor-induced proteinases. $J$ Cell Biol 1989 ; 108 : 671-82.

11. Bashkin $\mathrm{P}$, Doctrow $\mathrm{S}$, Klagsbrun $\mathrm{M}$ Svan CM, Folkman J, Vlodavsky I. Basic fibroblast growth factor binds to subendothelial extra-cellular matrix and is released by heparinase and heparinlike molecules. Biochemistry 1989; 28 : 1737-43.

12. Folkman J. Angiogenesis. In : Verstraete M, Vermylen J, Lijnen R, Arnout J, eds : Thrombosis and Haemostasis, Leuven : Leuven University Press, 1987 : 583-96.

13. Ingber DE, Folkman J. How doses extracellular matrix control capillary mor-

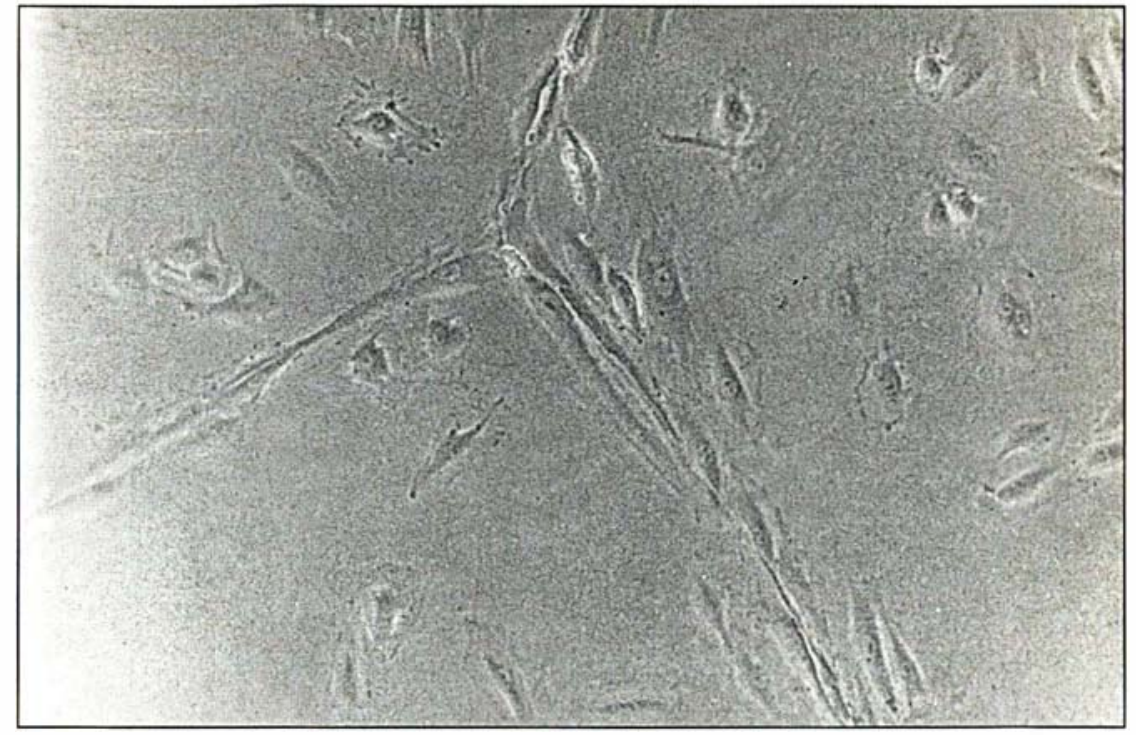

Figure 2. Élongation de cellules endothéliales humaines en culture et ébauche de formation de tubes sur matrice de gélatine $(x 50)$.

- la croissance de la plupart des tumeurs solides est dépendante de l'angiogenèse ;

- l'angiogenèse tumorale est induite directement ou indirectement par la tumeur elle-même.

En 1984, Judah Folkman écrivait : "Once tumor take has occurred, every increase in tumor cell population must be preceded by an increase in new capillaries that converge upon the tumor " [14]. Cette phrase résume parfaitement la dépendance tumorale vis-à-vis de l'angiogenèse. Un foyer métastatique privé de sa néovascularisation arrête sa croissance dès que le volume tumoral est de 1 à $2 \mathrm{~mm}^{3}$. Si la formation de néovaisseaux est restaurée, la croissance tumorale redémarre. $\mathrm{La}$ distance maximale entre un foyer tumoral et le lit capillaire néoformé peut être de 150 à $200 \mu \mathrm{m}$, distance qui permet encore la diffusion de l'oxygène. L'activité mitotique des cellules tumorales au sein d'un foyer métastatique diminue au fur et à mesure qu'augmente la distance de ces cellules aux capillaires les plus proches [1].

L'induction d'une angiogenèse à partir de facteurs diffusibles d'origine tumorale est une donnée qui a été pour la première fois évoquée il y a plus de vingt ans [15]. Dans les années 70 , des purifications partielles de facteurs angiogéniques ont pu être réalisées à partir de broyat de tumeurs ou de milieux conditionnés de cultures de cellules transformées [16]. Mais c'est surtout à partir des années 80 que l'explosion des connaissances sur les facteurs de croissance va permettre une caractérisation plus précise des substances angiogéniques (voir [1] pour revue). L'angiogenèse tumorale peut aussi être induite indirectement par la tumeur. Les macrophages présents dans un foyer tumoral sécrètent des facteurs capables de stimuler l'angio- 
genèse in vitro et in vivo $[17,18,19]$. C'est l'activation de ces macrophages par des stimuli d'origine tumorale qui serait responsable de leur sécrétion de facteurs angiogéniques. Enfin, comme nous l'avons déjà évoqué, des enzymes (collagénases, héparanases) pourraient être sécrétées par les cellules tumorales; leur action sur la matrice entraînerait la libération de facteurs de croissance pour les cellules endothéliales [11].

L'angiogenèse tumorale peut donc être en rapport avec un ou plusieurs mécanismes (figure 3). Elle assure la croissance tumorale et le développement des métastases. Récemment, deux découvertes importantes sont venues compléter l'ensemble des données descriptives sur l'angiogenèse tumorale. Folkman et al. [20] viennent de montrer, grâce à un modèle de souris transgéniques qui expriment un oncogène dans les cellules $\beta$ des îlots pancréatiques, que la progression "de l'état hyperplasie à l'état néoplasie" est précédée par l'apparition d'une forte activité angiogénique. Cette découverte suggère que l'angiogenèse est une étape importante de la carcinogenèse. Ces résultats sont peut-être à rapprocher des travaux récents sur les gènes de répression tumorale. Rastinejad et al. [21] ont en effet identifié un inhibiteur puissant de l'angiogenèse d'un poids moléculaire de $140 \mathrm{kDa}$ dont l'arrêt de sécrétion par les cellules BHK coïncide avec la perte d'un gène de répression tumorale, avec la transformation cellulaire et avec l'acquisition d'une activité angiogénique.

\section{Les facteurs angiogéniques}

De nombreux facteurs de croissance sont susceptibles d'induire une angiogenèse in vitro et/ou in vivo (Tableau I, p. 430). Plusieurs d'entre eux ont pu être purifiés, séquencés et clonés. Il est ainsi apparu que la plupart de ces facteurs étaient également présents dans des cellules ou tissus non transformés [22]. Nous limiterons cette revue à certains facteurs angiogéniques en raison soit de leur importance, soit parce qu'ils font l'objet de travaux dans notre équipe.

$\mathrm{m} / \mathrm{s} n^{\circ} 5 \mathrm{rol}$. 6, mai 90

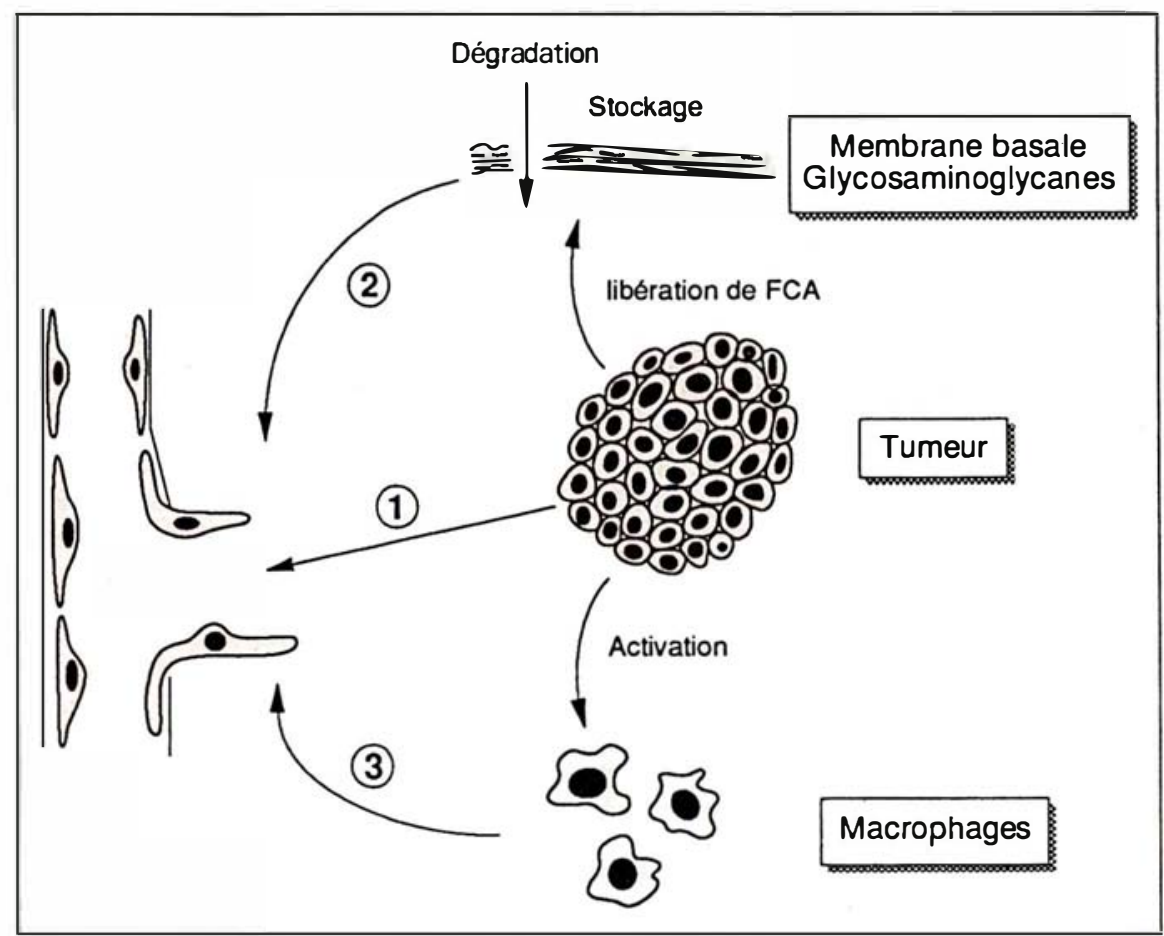

Figure 3. Angiogenèse tumorale. 1. Par libération directe de facteurs de croissance et angiogéniques (FCA). 2. Par dégradation de composants matriciels ayant stocké des FCA. 3. Par activation des macrophages avec libération secondaire de FCA.

Les facteurs de croissance des fibroblastes (FGF)

Ces facteurs sont aussi appelés " heparin binding growth factors" (HBGF) en raison de leur forte affinité pour l'héparine. Ils sont classés en deux groupes: $\mathrm{FGF}$ acide ou $\mathrm{HBGF}_{1}$, et FGF basique ou $\mathrm{HBGF}_{2}$ (voir [23, 24] pour revue). Les $\mathrm{FGF}$ ont pu être isolés à partir de nombreuses cellules et tissus normaux ou transformés. Ils stimulent l'angiogenèse in vitro et in vivo. Le FGF basique est une protéine de $18 \mathrm{kDa}$, formée d'une chaîne polypeptidique de 155 acides aminés. Il ne possède pas de séquence " signal ". Les domaines de liaison à l'héparine ont pu être localisés. Il présente une homologie de $55 \%$ avec le FGF acide, mais leurs gènes respectifs sont différents. Le gène du FGF basique est localisé sur le chromosome 4, il est constitué de 3 exons et de 2 introns et code pour une protéine qui possède une taille supérieure à $37 \mathrm{kDa}$. L'espèce de
$18 \mathrm{kDa}$ est initiée par des codons AUG alors que des espèces alternatives de 20 à $24 \mathrm{kDa}$ semblent être initiées par des codons CUG. Différents produits d'oncogènes ont été identifiés comme étant homologues aux FGF (Tableau $I I$, p. 431). Des récepteurs spécifiques pour le FGF basique ont pu être mis en évidence sur les cellules endothéliales animales et humaines, macro - et microvasculaires [25]. On distingue des sites de haute affinité et des sites de basse affinité. Ces derniers sont très vraisemblablement des glycosaminoglycanes. Les cellules endothéliales ellesmêmes peuvent synthétiser le FGF basique. Plusieurs espèces d'ARNm sont transcrites à partir du gène dans les cellules endothéliales microvasculaires humaines [26]. En revanche, malgré la production de FGF basique à l'intérieur des cellules, nous n'avons pas pu mettre en évidence d'excrétion du facteur dans le milieu extracellulaire, probablement en raison de l'absence de séquence "si- 


\section{RÉFÉRENCES}

14. Folkman J. Angiogenesis. In : Jaffe EA, ed : Biology of Endothelial Cells, Boston : Martinus Nijhoff Publishers, 1984 : 412-28.

15. Folkman J, Merler E, Abernathy C, Williams G. Isolation of a tumor factor responsible for angiogenesis. J Exp Med 1971 ; 133 : 275-88.

16. Kull FC, Brent DA, Parikh I, Cuatrecasas $P$. Chemical identification of a tumorderived angiogenic factor. Science 1987 ; $236: 843-5$

17. Leibovich SJ, Polverini PJ, Shepard HM, Wiseman DM, Shively V, Nuseir N. Macrophage-induced angiogenesis is mediated by tumour necrosis factor $\alpha$. Nature $1987 ; 239: 630-2$.

18. Höckel M, Sasse J, Wissler J. Purified monocyte-derived angiogenic substance (angiotropin) stimulates migration, phenotypic changes, and " tube formation " but not proliferation of capillary endothelial cells in vitro. J Cell Physiol 1987; 133 : 1-3.

19. Höckel $M$, Jung $W$, Vaupel $P$, Rabes $\mathrm{H}$, Khaledpour C, Wissler JH. Purified monocyte-derived angiogenic substance (angiotropin) induces controlled angiogenesis associated with regulated tissue proliferation in rabbit skin. J Clin Invest 1988; 82 : 1075-90.

20. Folkman J, Watson $\mathrm{K}$, Ingber $\mathrm{D}$, Hanahan D. Introduction of angiogenesis during the transition from hyperplasia to neoplasia. Nature 1989 ; 339 : 58-61.

21. Rastinejad F, Polverini PL, Bouck NP. Regulation of the activity of new inhibitor of angiogenesis by a cancer suppressor gene. Cell 1989 ; 56 : 345-55.

22. Dreyfus JC. Les facteurs angiogéniques. médecine/sciences $1988: 5: 318-9$.

23. Lobb RR. Clinical applications of heparin-binding growth factors. Eur J Clin Invest $1988 ; 18$ : 321-36.

24. Rifkin DB, Moscatelli D. Recent developments in the cell biology of basic fibroblast growth factor. J Cell Biol 1989 ; 109 : $1-6$.

25. Bikfalvi A, Dupuy E, Fayien N, Lesèche G, Courtois Y, Tobelem G. Binding, internalization and degradation of basic fibroblast growth factor in human microvascular endothelial cells. Exp Cell Res 1989 ; 181: $75-84$

26. Bikfalvi A, Alterio J, Courtois J, Tobelem G. Expression of bFGF in human omental endothelial cells. $J$ Cell Physiol (in

\begin{tabular}{|l|c|c|}
\hline \multicolumn{3}{|c|}{ Tableau I } \\
PRINCIPAUX COMPOSÉS CAPABLES DE STIMULER \\
OU D'INHIBER LA CROISSANCE ET/OU LA DIFFÉRENCIATION \\
DES CELLULES ENDOTHÉLIALES \\
\hline Nom & Effet sur la & Effet sur la \\
& croissance & différenciation \\
\hline Angiogénine & 0 & + \\
Angiotropine & 0 & + \\
FGF acide & + & + \\
FGF basique & + & + \\
GM-CSF & + & $?$ \\
Héparine & ou & (avec stéroïdes) \\
IL-6 & - & $?$ \\
Interféron $\alpha$ & - & + \\
Protamine & - & + \\
Thrombine & + & + \\
TGF $\beta$ & - & + \\
TGF $\alpha$ & + & $?$ \\
TNF $\alpha$ & - & + \\
Vasculotropine & + & + \\
\hline
\end{tabular}

+ : effet stimulant; - : effet inhibiteur ; 0 : pas d'effet.

gnal ". Or, il est présent dans les matrices extracellulaires [11]. Ce dépôt extracellulaire de FGF pourrait être secondaire à une lyse des cellules endothéliales, à une cosécrétion avec des protohéparanes sulfates, à une phosphorylation intracellulaire post-traductionnelle ou à l'existence d'un transporteur spécifique non encore identifié $[11,24]$. Les FGF, facteurs fortement angiogéniques présents dans les macrophages, certaines cellules tumorales, les matrices et les cellules endothéliales, pourraient jouer un rôle important dans l'angiogenèse tumorale. Ce rôle, cependant, vient d'être mis en doute dans une série d'expériences récentes car des hybridomes sécrétant des anticorps anti-bFGF bloquants, implantés chez la souris nude, ont formé des tumeurs parfaitement vascularisées suggérant ainsi que le bFGF ne serait pas le facteur angiogénique de ce type de tumeur [27].

\section{L'angiogénine [28]}

C'est un polypeptide isolé à partir du milieu conditionné d'une lignée cel- lulaire d'adénocarcinome humain. Son poids moléculaire est de 14400 , elle présente une homologie de $35 \%$ avec les ribonucléases pancréatiques. En revanche, elle est totalement différente des HBGF et n'a aucune affinité pour l'héparine. Elle possède un peptide "signal " et est donc sécrétée par les cellules en culture, c'est une protéine circulante. L'originalité de son action réside dans le fait qu'elle est dépourvue d'activité mitogène pour les cellules endothéliales bien que des récepteurs viennent d'être récemment mis en évidence sur des cellules endothéliales capillaires [29]. Ses effets angiogéniques in vivo font donc très vraisemblablement intervenir soit une action sur la différenciation des cellules endothéliales, soit sur le recrutement d'autres cellules susceptibles de produire des facteurs mitogènes.

\section{L'angiotropine [18, 19]}

C'est un polyribonucléopolypeptide contenant du cuivre, isolé à partir de cultures de monocytes activés. Ce facteur également non mitogène est 
un puissant agent de différenciation des cellules capillaires; il induit la formation de tubes; il stimule aussi la migration des cellules endothéliales microvasculaires. Le recrutement des monocytes-macrophages au sein d'un foyer tumoral pourrait avoir pour conséquence une libération d'angiotropine et stimuler de la sorte l'angiogenèse.

\section{La vasculotropine [30]}

Ce facteur angiogénique est le dernier découvert. Il a été isolé à partir de cellules folliculaires de l'hypophyse de bœuf ou à partir du milieu conditionné de la lignée cellulaire hypophysaire AtT-20 (J. Plouët, communication personnelle). Il porte également le nom de folliculo stellatederived growth factor (FSdGF). Il s'agit d'un homodimère composé de deux sous-unités de poids moléculaire $23 \mathrm{kDa}$. C'est un mitogène très puissant pour les cellules endothéliales (actif à $50 \mathrm{pg} / \mathrm{ml}$ ). Il est très spécifique de celles-ci puisqu'il n'a aucune action mitogène sur une grande variété d'autres types cellulaires. Il ne présente aucune homologie avec d'autres protéines connues. Sa présence éventuelle dans des tissus ou des cellules transformées est actuellement recherchée. Son rôle dans l'angiogenèse tumorale n'est donc pas encore établi.
Le " transforming growth factor " [31]

Le TGF $\alpha$ qui présente une forte homologie avec l'epidermal growth factor stimule l'angiogenèse in vivo et est mitogène pour les cellules endothéliales. Le TGF $\beta$ entraîne in vivo, après injection sous-cutanée, une accumulation de macrophages, de fibroblastes et de collagène ; et induit une néovascularisation. En revanche, in vitro, il inhibe la croissance des cellules endothéliales. Son activité angiogénique in vivo met donc probablement en jeu les macrophages.

Les autres facteurs angiogéniques La thrombine $\alpha$ est fortement mitogène pour les cellules endothéliales macrovasculaires, elle pourrait également agir sur la différenciation des cellules endothéliales microvasculaires [32].

Le tumor necrosis factor $\alpha$ (TNF $\alpha)$ stimule la migration des cellules endothéliales et leur formation en tubes. L'angiogenèse induite par les macrophages pourrait être en partie reliée à leur capacité de sécrétion de TNF $\alpha$ [33].

Le tumor vascular permeability factor est une protéine d'un poids moléculaire de $40 \mathrm{kDa}$ purifiée à partir du milieu conditionné d'une lignée de " cellules tumorales 10 " de cobayes [34]. Ce facteur, initialement décrit comme

\begin{tabular}{|c|c|c|c|c|c|}
\hline \multicolumn{6}{|c|}{ Tableau II } \\
\hline Nom & Synonyme & $\begin{array}{l}\text { Séquence } \\
\text { " signal " }\end{array}$ & $\begin{array}{l}\text { Liaison à } \\
\text { l'héparine }\end{array}$ & $\begin{array}{l}\text { Localisation } \\
\text { chromo- } \\
\text { somique }\end{array}$ & $\begin{array}{c}\text { Homologie } \\
\text { au FGF } \\
\text { basique }\end{array}$ \\
\hline FGF-1 & $\begin{array}{l}\text { FGF acide/ } \\
\text { HBGF }_{1}\end{array}$ & 0 & + & 5 & $55 \%$ \\
\hline FGF-2 & $\begin{array}{c}\text { FGF basiquel } \\
\text { HBGF }_{2}\end{array}$ & 0 & + & 4 & $100 \%$ \\
\hline $\begin{array}{l}\text { FGF-3 } \\
\text { FGF-4 } \\
\text { FGF-5 } \\
\text { FGF-6 } \\
\text { FGF-7 }\end{array}$ & $\begin{array}{c}\text { hst/K-FGF } \\
\text { int-2 } \\
\text { KGF }\end{array}$ & $\begin{array}{l}+ \\
0 \\
+ \\
+ \\
+\end{array}$ & $\begin{array}{l}+ \\
+ \\
+ \\
\text { ND } \\
\text { ND }\end{array}$ & $\begin{array}{r}11 \\
11 \\
\text { ND } \\
12 \\
\text { ND }\end{array}$ & $\begin{array}{l}43 \% \\
44 \% \\
43 \% \\
\text { ND } \\
39 \%\end{array}$ \\
\hline
\end{tabular}

ND : non déterminé.

$\mathrm{m} / \mathrm{s} n^{\circ} 5$ vol. 6 , mai 90 augmentant la perméabilité vasculaire, est angiogénique in vivo et stimule la prolifération des cellules endothéliales en culture.

Il est difficile, parmi cette pléiade non exhaustive de facteurs angiogéniques, de désigner celui (ou ceux) qui joue(nt) un rôle important in vivo dans le développement de l'angiogenèse tumorale. Leur obtention à l'état d'homogénéité, la préparation d'anticorps spécifiques, leur clonage, devraient permettre de faire progresser nos connaissances. Les enjeux sont importants : l'angiogenèse pathologique doit pouvoir être inhibée, or, comme nous allons le voir, l'inhibition pharmacologique de l'angiogenèse n'en est qu'à ses premiers balbutiements; l'angiogenèse réparatrice doit pouvoir être stimulée, et tout est encore à faire dans ce domaine.

\section{Inhibition de l'angiogenèse}

Compte tenu de la dépendance de la croissance tumorale vis-à-vis de l'angiogenèse, le concept d'une thérapeutique anti-angiogénique est rapidement apparu très séduisant $[1,12]$. Les tissus avasculaires, comme le cartilage, ont été les premiers à faire l'objet d'une recherche d'un inhibiteur de l'angiogenèse. Des extraits de cartilage se sont effectivement montrés être anti-angiogéniques. Mais la purification des substances inhibitrices responsables n'a pas été poursuivie. Certains agents antiinflammatoires ont un effet antiangiogénique. La protamine est un bon inhibiteur de l'angiogenèse, mais son administration prolongée est toxique. Les travaux les plus intéressants concernent les héparines et les stéroïdes. Les héparines ont une action complexe sur la prolifération des cellules endothéliales. Elles stimulent leur croissance lorsque celle-ci est induite par le FGF acide. En eff et ce facteur acquiert, en présente d'héparine, une affinité plus grande pour son récepteur cellulaire. En revanche, comme nous l'avons montré, lorsque les cellules endothéliales sont préincubées avec l'héparine, leur croissance, normalement induite par l'addition secondaire de sérum ou de facteur de croissance, est fortement 


\section{RÉFÉRENCES}

27. Matsuzaki K, Yoshitake Y, Matuo Y, Sasaki H, Nishikawa K. Monoclonal antibodies against heparin-binding growth factor II/basic fibroblast growth factor that block its biological activity : invalidity of the antibodies for tumor angiogenesis. Proc Natl Acad Sci USA 1989 ; 86 : 9911-5.

28. Fett JW, Strydom DJ, Lobb R, et al Isolation and characterization of angiogenin an angiogenic protein from human carcinoma cells. Biochemistry $1985 ; 24$ : 5480-6.

29. Badet J, Soncin F, Guitton JD, Cart wright $T$, Barritault D. Specific binding of angiogenin to calf pulmonary artery endo thelial cells. Proc Natl Acad Sci USA 1989 ; 86 : 8427-31

30. Gospodarowicz D, Abraham JA, Schilling $\mathrm{J}$. Isolation and characterization of a vascular endothelial cell mitogen produced by pituitary-derived folliculo stellate cells. Biochemistry 1989 ; 86 : 7311-5.

31. Folkman J, Klagsbrun M. A family of angiogenic peptides. Nature 1987; 329 : $671-2$.

32. Dupuy E, Bikfalvi A, Rendu F, Levy Toledano S, Tobelem G. Thrombin mitogenic responses and protein phosphorylation are different in cultured human endothelia cells derived from large and microvessels. Exp Cell Res 1989 ; 85 : 363-72.

33. Dreyfus JC. Une protéine à facettes multiples : le facteur nécrosant des tumeurs (tumor necrosis factor, TNF). médecine/sciences $1988 ; 4: 651-4$

34. Conolly DT, Henvelman DM, Nelson $\mathrm{R}$, et al. Tumor vascular permeability factor stimulates endothelial cell growth and angiogenesis. J Clin Invest 1989; 84 : 1470-8.

35. Rosenbaum J, Tobelem G, Molho P, Barzu T, Caen J. Modulation of endothelial cells growth induced by heparin. Cell Biol Int Rep 1986 ; 10 : 437-46.

36. Bikfalvi A, Dupuy E, Ruan C, Tobelem $G$, Lesèche $G$, Caen J. Binding of heparin to human microvascular endothelial cell and the effect on proliferation. Cell Biol Int Rep 1988; 12 : 931-42.

37. Crum R, Szabo S, Folkman J. A new class of steroids inhibits angiogenesis in the presence of heparin or a heparin fragment. Science 1985 ; 230 : 1375-8.

38. Cariou R, Harrousseau JL, Tobelem G. Inhibition of human endothelial cell proliferation by heparin and steroids. Cell Biol Int Rep 1988; 12 : 1037-47. inhibée [35]. Cette inhibition est en relation avec une action intracellulaire de l'héparine qui a été internalisée par les cellules endothéliales. Cette activité inhibitrice de l'héparine est observée avec des héparines de bas poids moléculaire et même avec le fragment héparinique pentasaccharidique, qui est totalement dépourvu d'activité anticoagulante [36]. Les concentrations néanmoins nécessaires sont importantes et suprapharmacologiques. Plus intéressants sont les effets anti-angiogéniques de l'association stéroïde/héparine. Crum et al. ont montré que des stéroïdes dépourvus d'activité minéralo- ou glucocorticoïde étaient de puissants inhibiteurs de l'angiogenèse in vivo, en présence d'héparine ou de fragments d'héparine [37]. Des régressions tumorales (M 5076, tumeur de Lewis, mélanome B16, MB 49) ont même été observées par ces auteurs chez la souris, après administration de cette association. Le mécanisme selon lequel l'angiogenèse est inhibée n'est pas établi avec certitude. Des altérations de la membrane basale pourraient être impliquées [37]. Nous avons, en ce qui nous concerne, mis en évidence un effet antimitotique synergique de l'association héparine/tétrahydroS sur des cellules endothéliales humaines en culture [38]. Les travaux sur ces stéroïdes anti-angiogéniques et sur les fragments d'héparine actifs se poursuivent, portant à la fois sur les mécanismes et sur l'expérimentation animale. Le maintien d'une tumeur ou sa régression, à un stade avasculaire c'est-à-dire de quelques $\mathrm{mm}^{3}$, représenterait un progrès considérable dans la stratégie thérapeutique anticancéreuse.

Depuis plus de vingt ans, le nom de Folkman est associé aux travaux portant sur l'angiogenèse. Aujourd'hui, de nombreuses équipes dans le monde consacrent leur recherche à ce domaine passionnant. La biologie cellulaire de l'angiogenèse a beaucoup progressé ; le phénomène est maintenant abordé au niveau biologie moléculaire. Les champs d'application d'une meilleure connaissance de l'angiogenèse sont vastes. Les médecins attendent beaucoup de ces progrès futurs

\section{Summary}

Tumor angiogenesis

The vascular system is lined by a monolayer of endothelial cells which proliferate very slowly in the normal conditions. A formation of new capillary vessels is associated with some physiological circumstances and several pathological conditions. Angiogenesis requires migration, differentiation and proliferation of endothelial cells. The mechanism of the tube formation is still poorly understood. Tumor growth is angiogenesis-dependent and angiogenesis is directly or indirectly induced by the tumor. Induction of angiogenesis is an important step of carcinogenesis and of metastatic development. Numerous angiogenic factors have been identified, most are mitogenic for endothelial cells and some are only responsible for the tube formation. Inhibition of tumor angiogenesis under the control of a tumor suppressor gene could play an important role. Pharmacological compounds such as heparin, heparin fragments and steroids have been shown to be antiangiogenic substances.

\section{TIRÉS A PART}

G. Tobelem. 